\title{
Weekly variations in feelings of trust predict incident STI within a prospective cohort of adolescent women from a US city
}

\author{
Pamela A Matson, ${ }_{5}$ J Dennis Fortenberry, ${ }^{2}$ Shang-en Chung, ${ }^{3}$ Charlotte A Gaydos, ${ }^{4}$ \\ Jonathan M Ellen ${ }^{5}$
}

\begin{abstract}
'Department of Pediatrics, Johns Hopkins School of Medicine, Baltimore, Maryland, USA ${ }^{2}$ Department of Pediatrics, Indiana University School of Medicine, Indianapolis, Indiana, USA

${ }^{3}$ Department of Medicine, Johns Hopkins School of Medicine, Baltimore, Maryland, USA ${ }^{4}$ Division of Infectious Diseases, Johns Hopkins University, Baltimore, Maryland, USA ${ }^{5} J o h n s$ Hopkins All Children's Hospital, St. Petersburg, Florida, USA
\end{abstract}

Correspondence to Dr Pamela A Matson, Department of Pediatrics, Johns Hopkins School of Medicine Baltimore, MD 21287, USA; pmatson@jhmi.edu

Received 10 October 2017 Revised 8 February 2018 Accepted 7 March 2018 Published Online First 24 March 2018
Check for updates

To cite: Matson PA, Fortenberry JD, Chung $S$, et al. Sex Transm Infect 2018:94:594-597.

\section{ABSTRACT}

Objectives Feelings of intimacy, perceptions of partner concurrency (PPC) and perceptions of risk for an STD (PRSTD) are meaningful and dynamic attributes of adolescent sexual relationships. Our objective was to examine whether variations in these STI-associated feelings and perceptions predicted incident Chlamydia trachomatis and/or Neisseriagonorrhoeae infection within a prospective cohort of urban adolescent women. Methods A cohort of clinic-recruited women aged 16-19 completed daily surveys on feelings and risk perceptions about each current sex partner on a smartphone continuously for up to 18 months. Urine was tested for $C$. trachomatis and $N$. gonorrhoeae every 3 months. Daily responses were averaged across the week. As overall means for trust, closeness and commitment were high, data were coded to indicate any decrease in feelings from the previous week. PRSTD and PPC were reverse coded to indicate any increase from the previous week. An index was created to examine the cumulative effect of variation in these feelings and perceptions. Generalised linear models were used to account for correlation among repeated measures within relationships.

Results For each week that there was a decrease in trust, there was a $45 \%$ increase in the risk of being infected with an STI at follow-up (relative risk (RR) 1.45, $95 \% \mathrm{Cl} 1.18$ to $1.78, \mathrm{P}=0.004)$. Neither a decrease in closeness or commitment, nor an increase in PRSTD or PPC was associated with an STI outcome. Cumulatively, the index measure indicated that a change in an additional feeling or perception over the week increased the odds of an STI by $14 \%$ (RR $1.14,95 \%$ Cl 1.02 to 1.29, $\mathrm{P}=0.026$ ).

Conclusions A decrease in feelings of trust towards a main partner may be a more sensitive indicator of STI risk than PRSTD, PPC or commitment. The next generation of behavioural interventions for youth will need strategies to address feelings of intimacy within adolescent romantic relationships.

Romantic and sexual relationships contribute to healthy adolescent development, yet relationships also confer risk. ${ }^{1}$ Relationships are a common context for transmission of the STIs that occur at high rates among adolescents and young adults. $^{2}$ In particular, perceptions of a partner's behaviours-such as believing the partner has other partners-drives risk perception and protective behaviours such as condom use. ${ }^{3} 4$ However, relationships are not exclusively focused on potential health risks, and relationship qualities such as feelings of intimacy are also key determinants of both adolescent risk perception and condom use. ${ }^{5}$ Adolescents consider intimacy as an essential feature of an ideal romantic relationship. ${ }^{67}$ Moving towards intimate relationships is a key developmental task of late adolescence ${ }^{1}$ and having an intimate partner is highly valued by adolescents. ${ }^{8}$

Intimacy is most often characterised as containing elements of trust, closeness and commitment. ${ }^{9} 10$ However, feelings of trust, closeness and commitment have been found to be inversely associated with condom use. ${ }^{11} 12$ Adolescents report lower levels of condom use in partnerships characterised by trust and commitment. ${ }^{9} 10$ Main partnership classifications (eg, boyfriend, steady partner, exclusive) signify the importance of the relationship and reflect levels of commitment and trust within the relationship. ${ }^{101113}$ Cessation of condom use within a relationship may signal advancing levels of intimacy in a relationship and serve to confirm intimacy by their absence. ${ }^{14}$

Despite its centrality in sexual and romantic relationships, adolescents' experiences of intimacy are not well understood. Intimacy is usually conceptualised as a static relationship quality, ${ }^{15}$ rather than one subject to variability of feelings, interactions and circumstances. Using daily data collected from adolescent and young women, our prior work demonstrates that trust and closeness are dynamic and variable within adolescent relationships. ${ }^{16}$ While adolescents overall report strong feelings of trust and closeness for a partner, there are significant day-to-day fluctuations in these feelings. In contrast, partner-specific perception of STI risk was found to be stable within a relationship. We hypothesised that fluctuations in feelings of intimacy are a more sensitive indicator of condom use and STI acquisition than a single static measure of intimacy.

The objective of this study, then, was to predict adolescent women's incident Chlamydia trachomatis or Neisseria gonorrhoeae infections based on daily variations in intimacy, perceptions of partner concurrency and STI risk perception. We hypothesised that changes in feelings of trust and closeness for a partner would more strongly predict 
incident STI than changes in perceptions of partner concurrency or STI risk perception. In order to capture the true extent of the variability, we used daily reports of partner-specific STI risk perception, perception of partner concurrency and feelings of trust, closeness and commitment.

\section{MATERIALS AND METHODS}

A detailed description of methods and cohort characteristics for the Perceived Risk for Sexually Transmitted Diseases (PRSTD) study has been published previously. ${ }^{16}$ The study procedures are described briefly below.

\section{Study population}

A prospective cohort of adolescent women was recruited from a hospital-based adolescent medicine clinic, a public STD clinic and community venues in Baltimore, Maryland, USA. Adolescent women were enrolled between December 2009 and August 2010. Eligibility criteria included age 16 to 19 years, sexually active defined as having vaginal or anal intercourse with an opposite sex partner in the preceding 3 months, English-speaking and residence in the Baltimore metropolitan area. Sixty-two per cent $(122 / 196)$ of eligible women agreed to participate. There were no differences in demographic or behavioural characteristics between participants and non-participants, with the exception of lifetime number of sex partners. Non-participants reported a greater mean lifetime sexual partners than participants $(5.5$ vs 2.0 , respectively, $\mathrm{P}<0.05)$. The Johns Hopkins University Institutional Review Board approved the study protocol (NA_00017298).

\section{Procedures}

Participants completed an audio computer-assisted self-interview (ACASI) at baseline and every 3 months. Daily questionnaires were completed using a Palm Centro smartphone continuously for 18 months. Every day, adolescents reported on their partner-specific feelings and perceptions. Participants entered the nickname or initials of each of their current sex partners. Partner initials were confirmed at regular intervals to ensure the same partner was followed over time. Participants' urine samples were collected at each quarterly follow-up interview and tested for C. trachomatis and N. gonorrhoeae using nucleic acid amplification test (NAAT). ${ }^{17}$ Participants received a voice and text plan as remuneration for completing the daily diaries and US $\$ 25$ for each ACASI interview.

\section{Measures}

For each partner named on a given day, participants were asked, "How close do you feel toward him today?", "How much do you trust him today", "How committed do you feel to him today?" and "If you were to have sex with him today without using a condom, how likely are you to get an STD from him?". Possible responses were not at all, not very, somewhat and very, which we quantified on an ordinal scale represented from 1 (low) to 4 (high). PRSTD response options were a five-item Likert scale (not at all likely, not very likely, somewhat likely, very likely, extremely likely). Our measure of perceived partner concurrency (PPC) was "How certain are you about whether he has other sex partners?" with response of extremely, very, a little and not much. If participants reported having sex with this partner on a given day, they were then asked "Did you use a condom?" with response of yes or no.

\section{Statistical analysis}

Incident STI, defined as testing positive for either C. trachomatis and/or N. gonorrhoeae at each quarterly follow-up visit, was the primary outcome of interest for this analysis. Cumulative incidence was determined by calculating the proportion of participants who tested positive for an STI out of the total number of participants who remained in the study and were tested at each quarterly follow-up period. Daily responses were averaged across the week. As means for feelings of trust, closeness and commitment were high in these relationships, data were coded to indicate any decrease in feelings from the previous week. PRSTD and PPC were reverse coded to indicate any increase in feelings from the previous week. In order to examine the cumulative effect of variation in these feelings and perceptions, we created an index measure by adding all the aforementioned variables together. Generalised linear models, specifically log-binomial regressions, were used to calculate risk ratios while accounting for the correlation among repeated measures within relationships. ${ }^{18}$ These models are robust to both unbalanced and missing data. ${ }^{19}$ All analyses were conducted using SAS (V.9.2; SAS Institute, Cary, North Carolina, USA).

\section{RESULTS}

Participants completed a total of 4969 partner-specific diary entries. Participants completed daily diaries on a mean (SD) of 2.6 (2.4) unique sex partners over the 18 months of data collection. ${ }^{16}$ Participant characteristics at baseline are shown in table 1. Participants were on average (SD) 18.4 (1.1) years old; 92\% were African American. The mean (SD) age at first sex was

Table 1 Baseline demographic and relationship characteristics of 122 female participants

\begin{tabular}{|c|c|c|c|c|}
\hline Characteristic & Total sample & STI negative ${ }^{*}(n=95)$ & STI positive* $(n=22)$ & $P$ value \\
\hline Age in years, mean (SD) & $18.4(1.1)$ & 18.4 & 18.4 & 0.94 \\
\hline African-American race, $\mathrm{n}(\%)$ & $112(92)$ & $93 \%$ & $91 \%$ & 0.78 \\
\hline Age at first sex, mean (SD) & $13.9(3.3)$ & 13.8 & 14.0 & 0.80 \\
\hline Maternal education <high school, n(\%) & $79(65)$ & $67 \%$ & $64 \%$ & 0.74 \\
\hline Lifetime no of sexual partners, mean (SD); median & $8(11) ; 5$ & 7.6 & 7.3 & 0.91 \\
\hline No of sexual partners in the past 3 months, mean (SD); median & $2(3) ; 1$ & 2.0 & 1.9 & 0.86 \\
\hline Condom use at first sex with partner, $\mathrm{n}(\%)$ & $83(73)$ & $72 \%$ & $81 \%$ & 0.42 \\
\hline Condom use at last sex with partner, $\mathrm{n}(\%)$ & $53(47)$ & $44 \%$ & $57 \%$ & 0.27 \\
\hline STD history, $\mathrm{n}(\%)$ & $67(55)$ & $53 \%$ & $68 \%$ & 0.19 \\
\hline Length of relationship in months, mean (SD); median & $17.5(20.0) ; 13$ & 17.8 & 17.5 & 0.97 \\
\hline
\end{tabular}

*Participants were tested for $C$. trachomatis and $N$. gonorrhoeae at baseline. 
Table 2 Unadjusted* risk ratio $(95 \% \mathrm{CI})$ of condom use and STI

\begin{tabular}{|c|c|c|c|c|}
\hline & \multicolumn{2}{|c|}{ Risk ratio of condom use } & \multicolumn{2}{|l|}{ Risk ratio of STIt } \\
\hline & $95 \% \mathrm{Cl}$ & $P$ value & $95 \% \mathrm{Cl}$ & $P$ value \\
\hline Trust & 0.96 (0.84 to 1.11$)$ & 0.587 & 1.45 (1.18 to 1.78$)$ & 0.0004 \\
\hline Closeness & 0.90 (0.77 to 1.05 ) & 0.168 & 1.38 (0.98 to 1.96$)$ & 0.067 \\
\hline Commitment & 0.92 (0.79 to 1.06$)$ & 0.229 & 1.23 (0.92 to 1.64$)$ & 0.159 \\
\hline Perceived partner concurrency & 0.83 (0.67 to 1.01$)$ & 0.069 & $1.10(0.86$ to 1.40$)$ & 0.459 \\
\hline Perceived risk for STDs & 0.93 (0.79 to 1.11$)$ & 0.419 & 1.33 (0.80 to 2.20$)$ & 0.274 \\
\hline Index & 0.95 (0.88 to 1.02$)$ & 0.154 & $1.14(1.02$ to 1.29$)$ & 0.026 \\
\hline
\end{tabular}

*Inferences did not change after controlling for index concurrency or partner age difference $>2$ years.

†C. trachomatis and/or N. gonorrhoeae infection.

13.9 (3.3) years old. Sixty-five per cent of participants reported that their mother had less than or equal to a high school education. The mean (SD) number of sexual partners in the 3 months preceding the baseline interview was 2 (3), the mean (SD) number of lifetime sexual partners was 8 (11) and 55\% of the sample reported ever having had an STD. Condom use at first and last sex with most recent main partner was $73 \%$ and $47 \%$, respectively. The mean (SD) length of these relationships at baseline was 17.5 (20.0) months (median, 13 months) and ranged from 0 to 97 months. The period of time observed for each relationship (based on diary data) was on average (SD) 26.6 (21.0) weeks long and ranged from 1 to 79 weeks or average days $=186$ (SD 147); range, 2-550 days. There were no differences in demographic or relationship characteristics between participants who tested positive for C. trachomatis and/or N. gonorrhoeae infection at baseline (table 1), nor between those who tested positive at the 3-month follow-up visit (data not shown).

Table 2 presents the unadjusted risk ratio for the association between weekly changes in feelings of trust, closeness, commitment, perceived partner concurrency, perceived risk for an STI and condom use at next sex. There were no statistically significant associations between trust, closeness, commitment, perception of partner concurrency or perceived risk for an STI and condom use at next sex. Inferences did not change after controlling for index concurrency or partner age difference of greater than 2 years.

The STI incidence (C. trachomatis and/or N. gonorrhoeae infection combined) was $16 \%$ at 3 months, $17 \%$ at 6 months, $8 \%$ at 9 months, $13 \%$ at 12 months, $13 \%$ at 15 months and $4 \%$ at 18 months. Table 2 presents the unadjusted risk of having an incident STI at each quarterly follow-up visit. For each week that there was a decrease in trust, there was a $45 \%$ increase in the risk of being infected with an STI at follow-up (relative risk (RR) $1.45,95 \%$ CI 1.18 to $1.78, \mathrm{P}=0.004)$. For each week that there was a decrease in closeness, there was a $38 \%$ increase in the risk of being infected with an STI at follow-up (RR 1.38, 95\% CI 0.98 to $1.96, \mathrm{P}=0.067)$; however, this did not reach significance at $\mathrm{P}<0.05$. Neither an increase in PRSTD or PPC nor a decrease in commitment was associated with an incident STI outcome. The index measure created to examine the cumulative effect of variation in these feelings and perceptions found that a change in an additional feeling or perception that week increased the risk of an STI by 14\% (RR 1.14, 95\% CI 1.02 to $1.29, \mathrm{P}=0.026)$.

\section{DISCUSSION}

Subjective relationship experiences have significant public health relevance, at least in terms of STI risk. Results of this study support our hypothesis that a decrease in feelings of trust towards a main partner was a more sensitive predictor of incident N. gonorrhoeae and C. trachomatis infection than STI risk perception, PPC or relationship commitment. While aspects of intimacy (ie, trust, closeness, commitment) are related, we found that they are also distinguishable qualities. Weekly variations in trust emerged as the strongest predictor of incident STI infection; however, the data suggested that changes in feelings of closeness are also informative.

We did not find an association between weekly variation in trust, closeness, commitment or STI risk perception and condom use. Ewing and Bryan similarly did not find an association between trust and condom use. ${ }^{20}$ These findings are not surprising given our understanding of how condoms are abandoned in main relationships. ${ }^{21}$ Previous research has found that young women's decisions to have sex with a partner is based on trust and not health risks. ${ }^{14}$ Finding that an increase in perceived partner concurrency was not associated with condom use may seem in contrast to expectations; however, it may support what some adolescents have reported in interviews that engaging in unsafe sex is used as a strategy to hold on to non-monogamous partners and reinforce intimacy in their relationship. ${ }^{22} 23$

Adolescents and young adults place great value on being in an intimate relationship. ${ }^{81024}$ Feelings of trust and closeness are associated with fidelity. If the goal is to maintain an intimate partnership, then there exists a real tension between the intimacy brought about by abandoning condom use within a relationship and the knowledge and awareness that a partner has other sex partners. Sex without a condom reinforces the trust, closeness and commitment to the relationship. ${ }^{25}$ Preserving intimacy in the relationship may overshadow concerns for STI risk, as resuming condom use with a partner implies infidelity. ${ }^{26}$

However, the broader context in which these relationships take place move considerations of health behaviour beyond expectations of monogamy. In socioeconomically disadvantaged settings, adolescent and young adult women do not have the luxury of monogamy. ${ }^{22} 232527$ These young women have to reconcile their romantic ideal within a context of constrained resources, specifically, having to share partners because partners themselves are a limited resource. Young women adapt to these circumstances by trusting that their main partner will be respectful enough to hide side partners as well as use condoms with side partners. ${ }^{28}$ In settings with limited resources, adolescents do not have many things to exchange, thus, it is logical that trust becomes a highly symbolic gesture and foundational for the maintenance of relationships. By extension, condoms become a physical symbol of this monogamy narrative, where use of condoms acknowledges a partner having other partners. ${ }^{22}$ In these ways, trust represents both relationship security, where there is no risk to having unprotected sex, and a symbolic practice, where not requesting condom use avoids threatening the relationship. ${ }^{15}$

Much of our understanding on the role of trust, closeness and commitment on condom use comes from qualitative interviews with 
adolescents. While trust has become a more prevalent construct in the literature with regard to STI testing and concurrency, it is rarely examined. The current study begins to quantify how feelings of trust and closeness are a barometer of both health behaviour and risk. A strength of this study is the longitudinal design, which provides the temporal ordering of feelings and perceptions and subsequent infection. Specifically, the daily data allowed us to quantify how variations in trust within a relationship are associated with STI risk. While there are many strengths to this study, findings should be interpreted in light of several limitations. A single item indicator was used to measure trust; however, we know that trust is multidimensional. ${ }^{29}$ Future work would be well served to further unpack trust, with a particular eye towards understanding how adolescents come to understand the currency of trust despite limited relationship experience. Our intensive within-relationships data collection was balanced by a smaller sample size, which may have resulted in less precise effect estimates as well as limited our ability to stratify the data. While these are preliminary findings, they support a foundation, which establishes the importance of feelings of intimacy when designing STI/HIV prevention programmes for adolescents. Lastly, these data are from a clinic sample of women who tend to be at higher risk for STI compared with school-based or other noncare-seeking samples. However, we do not have reason to believe there are systematic differences in the association between feelings of intimacy and STI incidence for care-seeking versus non careseeking youth. Our sample represents the population who bear the greatest burden of STI, African-American adolescents and young adults. We do not mean to suggest that our findings will be generalisable to suburban or rural African Americans or to youth of other race/ethnicities; however, our findings should be relevant to other African-American urban populations.

These data illustrate the importance of emotional aspects of adolescent relationships and that feelings of trust for a partner cannot be underestimated. These findings underscore the importance of understanding the context of adolescent romantic relationships and how the intrinsic value of relationships may undermine intervention and prevention efforts. The next generation of behavioural interventions should consider strategies that leverage these positive relationship attributes.

\section{Key messages}

- Adolescents' experiences of intimacy are of great importance in their sexual and romantic relationships, but they are not well understood.

- A change in feelings of trust for a partner predicted incident STIs.

- The next generation of behavioural interventions should consider strategies that leverage positive relationship qualities.

\section{Handling editor Adam Huw Bourne}

Contributors PAM and JME collaborated on the research question and study design. PAM, JME and SC chose the main directions for data analysis and interpreted results. PAM prepared the manuscript. SC performed the statistical analyses. CAG conducted the laboratory testing. JDF added to the interpretation of results and edited the manuscript. All authors commented on drafts and approved the final manuscript.

Funding This study was supported by the National Institute of Child Health and Human Development (NICHD R01HD058309; PI: Ellen) and the National Institute of Drug Abuse (NIDA K01DA035387; PI: Matson).

Competing interests None declared.

Ethics approval Johns Hopkins University.
Provenance and peer review Not commissioned; externally peer reviewed.

Data sharing statement Requests for data sharing can be made to the first author.

(C) Article author(s) (or their employer(s) unless otherwise stated in the text of the article) 2018. All rights reserved. No commercial use is permitted unless otherwise expressly granted.

\section{REFERENCES}

1 Collins WA, Welsh DP, Furman W. Adolescent romantic relationships. Annu Rev Psychol 2009;60:631-52.

2 Centers for Disease Control and Prevention. STDs in adolescents and young adults. 2014.

3 Ellen JM, Adler N, Gurvey JE, et al. Adolescent condom use and perceptions of risk for sexually transmitted diseases: a prospective study. Sex Transm Dis 2002;29:756-62.

4 Gurvey JE, Adler N, Ellen JM. Factors associated with self-risk perception for sexually transmitted diseases among adolescents. Sex Transm Dis 2005;32:742-4.

5 Matson PA, Chung SE, Sander P, et al. The role of feelings of intimacy on perceptions of risk for a sexually transmitted disease and condom use in the sexual relationships of adolescent African-American females. Sex Transm Infect 2012;88:617-21.

6 Fletcher GJ, Simpson JA, Thomas G. Ideals, perceptions, and evaluations in early relationship development. J Pers Soc Psychol 2000;79:933-40.

7 Overbeek G, Ha T, Scholte R, et al. Brief report: Intimacy, passion, and commitment in romantic relationships - validation of a 'triangular love scale' for adolescents. J Adolesc 2007;30:523-8.

8 Volpe EM, Morales-Alemán MM, Teitelman AM. Urban adolescent girls' perspectives on romantic relationships: initiation, involvement, negotiation, and conflict. Issues Ment Health Nurs 2014;35:776-90.

9 Towner SL, Dolcini MM, Harper GW. Romantic relationship dynamics of urban African American adolescents: patterns of monogamy, commitment, and trust. Youth Soc 2015;47.

10 Bauman LJ, Berman R. Adolescent relationships and condom use: trust, love and commitment. AIDS Behav 2005;9:211-22

11 Manning WD, Flanigan CM, Giordano PC, et al. Relationship dynamics and consistency of condom use among adolescents. Perspect Sex Reprod Health 2009;41:181-90.

12 Sayegh MA, Fortenberry JD, Shew M, et al. The developmental association of relationship quality, hormonal contraceptive choice and condom non-use among adolescent women. J Adolesc Health 2006;39:388-95.

13 Ellen JM, Cahn S, Eyre SL, et al. Types of adolescent sexual relationships and associated perceptions about condom use. J Adolesc Health 1996;18:417-21.

14 Mullinax M, Sanders S, Higgins J, et al. Establishment of safety paradigms and trust in emerging adult relationships. Cult Health Sex 2016;18:890-904.

15 Willig C. The limitations of trust in intimate relationships: constructions of trust and sexual risk taking. Br J Soc Psychol 1997;36:211-21.

16 Matson PA, Chung SE, Huettner S, et al. Understanding variability in adolescent women's sexually transmitted infection-related perceptions and behaviors associated with main sex partners. Sex Transm Dis 2014;41:475-9.

17 Gaydos CA, Quinn TC. Urine nucleic acid amplification tests for the diagnosis of sexually transmitted infections in clinical practice. Curr Opin Infect Dis 2005; 18:55-66.

18 McNutt LA, Wu C, Xue $X$, et al. Estimating the relative risk in cohort studies and clinical trials of common outcomes. Am J Epidemiol 2003;157:940-3.

19 Ibrahim JG, Chen M-H, Lipsitz SR, et al. Missing-data methods for generalized linear models. J Am Stat Assoc 2005; 100:332-46.

20 Ewing SW, Bryan AD. A question of love and trust? the role of relationship factors in adolescent sexual decision making. J Dev Behav Pediatr 2015;36:628-34.

21 Fortenberry JD, Tu W, Harezlak J, et al. Condom use as a function of time in new and established adolescent sexual relationships. Am J Public Health 2002;92:211-3.

22 Sobo EJ. Inner-city women and AIDS: the psycho-social benefits of unsafe sex. Cult Med Psychiatry 1993;17:455-85.

23 Jones R, Oliver M. Young urban women's patterns of unprotected sex with men engaging in HIV risk behaviors. AIDS Behav 2007;11:812-21.

24 Carver K, Joyner K, Udry JR. National estimates of adolescent romantic relationships. Adolescent romantic relations and sexual behavior: theory, research, and practical implications. Mahwah, NJ: Lawrence Erlbaum Associates Publishers, 2003:23-56.

25 Eyre SL, Flythe M, Hoffman V, et al. Concepts of infidelity among African American emerging adults: implications for HIV/STI prevention. J Adolesc Res 2011;27:231-55.

26 Gebhardt WA, Kuyper L, Greunsven G. Need for intimacy in relationships and motives for sex as determinants of adolescent condom use. J Adolesc Health 2003;33:154-64

27 Matson PA, Chung SE, Ellen JM. Perceived neighborhood partner availability, partner selection, and risk for sexually transmitted infections within a cohort of adolescent females. J Adolesc Health 2014;55:122-7.

28 Andrinopoulos K, Kerrigan D, Ellen JM. Understanding sex partner selection from the perspective of inner-city black adolescents. Perspect Sex Reprod Health 2006;38:132-8.

29 Rempel JK, Holmes JG, Zanna MP. Trust in close relationships. J Pers Soc Psychol 1985:49:95-112. 